Questions de communication

\title{
Jacques LE BOHEC, Sociologie du phénomène Le Pen
}

Paris, Éd. La Découverte, coll. Repères, 2005, 122 p.

\section{Laurent Trémel}

\section{OpenEdition}

\section{Journals}

\section{Édition électronique}

URL : http://journals.openedition.org/questionsdecommunication/7426

DOI : 10.4000/questionsdecommunication.7426

ISSN : 2259-8901

\section{Éditeur}

Presses universitaires de Lorraine

\section{Édition imprimée}

Date de publication : 1 juillet 2007

ISBN : 978-2-86480-829-9

ISSN : 1633-5961

\section{Référence électronique}

Laurent Trémel, « Jacques Le вонес, Sociologie du phénomène Le Pen», Questions de communication [En ligne], 11 | 2007, mis en ligne le 01 juillet 2007, consulté le 12 avril 2021. URL : http:// journals.openedition.org/questionsdecommunication/7426 ; DOI : https://doi.org/10.4000/ questionsdecommunication.7426

Ce document a été généré automatiquement le 12 avril 2021 


\title{
Jacques LE BOHEC, Sociologie du phénomène Le Pen
}

Paris, Éd. La Découverte, coll. Repères, 2005, 122 p.

\author{
Laurent Trémel
}

\section{RÉFÉRENCE}

Jacques LE BOHEC, Sociologie du phénomène Le Pen. Paris, Éd. La Découverte, coll. Repères, 2005, $122 \mathrm{p}$.

1 Publié dans la collection "Repères ", ce petit livre se présente comme un ouvrage de synthèse permettant d'expliquer la place aujourd'hui accordée à Jean-Marie Le Pen, président du Front national (FN), dans la vie politique française. Il se compose de quatre chapitres. Le premier (pp. 11-31) est consacré à expliquer la percée médiatique et les premiers succès électoraux de l'homme politique au cours des années 80 , en incriminant d'emblée la responsabilité de François Mitterrand qui aurait pressé, dès 1982, les dirigeants de TF1 de faire œuvre de pluralisme en donnant la parole au leader de l'extrême droite. Dès lors, l'auteur n'est pas avare de jugements de valeur sur la personne de François Mitterrand, accusé d'avoir remis en place un «monarchisme présidentialiste» (p. 13) et d'avoir favorisé Jean-Marie Le Pen pour des raisons électoralistes (volonté de faire «oublier» des promesses électorales que la gauche ne tiendra pas en allumant des «contre-feux»). L'ex-président de la République est qualifié de «manœuvrier et cynique» (p. 14) et, après avoir rappelé la «jeunesse française » du chef de file des socialistes, il l'accuse d'avoir passé un accord implicite avec le leader du FN pour générer du trouble dans la vie politique de notre pays. Ayant réussi à s'attirer l'attention des médias, Jean-Marie Le Pen remplit en quelque sorte son "rôle », et les - relatifs - succès électoraux du FN de 1984 sont présentés comme une suite logique de la manœuvre amorcée en 1982.C'est alors «l'appareil» du Parti socialiste qui est incriminé : "Confirmant l'intérêt objectif de l'exécutif socialiste à diviser la coalition de droite en aidant le FN, c'est au début du mois de septembre 1984 
que Laurent Fabius estime que Jean-Marie Le Pen "pose de bonnes questions". Autrement dit, c'est le Premier ministre, avec son autorité statutaire, qui lui donne raison. De plus, en tant qu'énarque d'origine juive, il est censé être à la fois compétent et sincère, apte à établir un état des lieux exact de la société française et incapable d'une collusion avec un leader suspecté d'antisémitisme » (p. 29).

2 Le second chapitre (pp. 32-49) est consacré à la «trajectoire sociale » de Jean-Marie Le Pen. L'universitaire indique ici qu'il fera preuve d'un souci d'objectivité, reprochant à nombre d'auteurs s'étant penchés sur la biographie du leader du FN d'avoir réinterprété sa vie dans une perspective positive ou négative, en fonction des événements du moment (sur ce point, il se réfère à la formule de Pierre Bourdieu (Actes de la recherche en sciences sociales, 62-63, juin 1986, pp. 69-72) de "L'illusion biographique ». Remontant à la prime enfance du petit Jean-Marie, il explique que celui-ci a été élevé dans une famille bretonne un peu particulière : enfant unique, dans un contexte prédominé par l'existence de familles nombreuses, Jacques Le Bohec interprète ce fait comme révélateur d'un « ethos petit-bourgeois » (p. 37) et d'un milieu miné par des désirs d'ascension sociale inassouvis qui seront reportés sur «Le Pen junior » (sic), chargé symboliquement de poursuivre les rêves de mobilité sociale de son père. Dans une perspective fortement teintée de références à Pierre Bourdieu, l'auteur passe donc en revue la scolarité de l'agent social, évoque son "activisme d'étudiant attardé et bohème » (p. 41) jusqu'à ses premiers engagements politiques aux côté des poujadistes (1958). Passant très rapidement sur la période 1962-1983, synonyme de «marginalité politique ", après avoir évoqué l'enrichissement providentiel de JeanMarie Le Pen (héritage Lambert) qui devient millionnaire, Jacques Le Bohec s'intéresse aux stratégies matrimoniales du personnage, confirmant selon lui son désir de " parvenir »: «sa première épouse vient de la bourgeoisie de province, la seconde appartient à la haute bourgeoisie » (p. 46). Il conclut ce chapitre en dressant un tableau de son «habitus» sous la forme de "dix prédispositions» (pp. 46-49) qu'il identifie comme: ambition, anticonformisme («mélange entre les habitudes du potache faluchard et du militaire de chambrée », p. 47), bagou, esprit gaulois (« en privé voire en public, J.-M. Le Pen ne rate pas souvent une occasion de lancer une blague à connotation sexuelle, voire scatologique, du genre [...]», ibid.), hexis corporelle (caractérisée par la mise en scène d'exploits physiques et de sa virilité), révolte, provocation, sens des différences de classe, sens du spectacle, vantardise (par exemple : "intérêt à susciter la rumeur pour se forger une réputation de gros dur", p. 48). L'exposé de ces traits de caractère est marqué par une certaine redondance, et l'on peut s'interroger sur la nature des travaux scientifiques ayant conduit à l'établissement de ces catégories ad hoc.

Le troisième chapitre - «Les explications proposées » (pp. 50-68) - vise à présenter un certain nombre de thèses permettant de comprendre le "phénomène Le Pen ». Là aussi, Jacques Le Bohec établit une typologie à partir de dix entrées qu'il qualifie «d'explications classiques» (pp. 50-59), en en montrant les apports et les limites: résurgence (de l'extrême droite), racisme, balancier (le FN serait censé « remplacer » le PC dans la vie politique), charisme, diversion-division (rappel de la stratégie mitterrandienne), médiatisation, symptôme (l'homme poserait les « bonnes questions » découlant de la situation de crise économique et politique), gaucho-lepénisme (vote Le Pen d'une partie de l'électorat de "gauche»), lepénisation des esprits, (National)populisme. En fait, l'auteur est plutôt critique face à ces explications, synthétisant les 
travaux de journalistes, d'analystes politiques ou d'universitaires. Il montre bien, notamment, comment certaines thèses "tendent à négliger leur propre concours à la construction symbolique du phénomène Le Pen » (p. 59) ou encore que celles-ci «très abstraites» (p. 68) et volontiers intellectualisantes, tendent «à se passer de contact direct et organisé avec des électeurs concrets » (ibid.).

Cette dernière assertion justifie le quatrième chapitre - "L'enquête auprès des électeurs» (pp. 69-94) -, rendant compte de travaux empiriques menés auprès de l'électorat de Jean-Marie Le Pen et du Front national. Celui-ci débute par deux parties : "Méthodes quantitatives » et "Méthodes qualitatives ». La première (pp. 71-76) dresse en fait un bilan plutôt critique des "sondages " effectués sur la question, montrant bien les biais méthodologiques engendrés par les usages ayant cours dans les instituts de sondage. Là aussi, il est reproché à ces travaux de concourir à la reproduction de certaines logiques: "Les enquêtés sont, par exemple, supposer accepter passivement des affirmations caricaturales qu'on leur demande d'approuver ou de désapprouver [...]» (p. 74) et Jacques Le Bohec insiste sur le caractère peu fiable de ces travaux où il relève des «impositions de problématiques» (pp. 71-74) ne permettant pas de réellement cerner les causes du vote Le Pen. A contrario, il présente quelques travaux scientifiques analysant plus finement (au niveau régional par exemple) les motivations de certains électeurs. La partie consacrée aux " méthodes qualitatives " s'intéresse là encore aux approches « profanes » et « savantes ». L'universitaire expose tour à tour les procédés utilisés par des journalistes et des chercheurs pour "s'infiltrer " parmi les électeurs lepénistes. Il y fait preuve d'un certain scepticisme quant aux apports de ces démarches, allant même jusqu'à écrire: "Il convient donc de mesurer la vanité de certains enquêteurs, tentés de se dépeindre en héros revenus d'un voyage dans une contrée dangereuse » (p. 78), De même, il se montre critique vis-à-vis des techniques de « reportage " plus banales (méthodes de questionnement discutables et échantillons biaisés aboutissant, là encore, à la production d'artefacts). In fine, il conclut en présentant les apports des entretiens (directifs et semi-directifs) que lui-même et d'autres chercheurs privilégient: "On obtient souvent, dans un simple entretien enregistré, une richesse et une fiabilité des informations que l'on cherche en vain dans un sondage " (p.81). Dans la dernière partie de ce chapitre - «Resituer la complexité » (pp. 83-94) -, Jacques Le Bohec aborde trois questions, présentées sous la forme de problématiques, lui paraissant essentielles lorsque l'on s'intéresse à l'électorat de JeanMarie Le Pen: "sens et usage du racisme " - en insistant notamment sur l'aspect provocateur que le fait de se déclarer " raciste" peut prendre chez certains électeurs du FN désillusionnés, dans un contexte marqué par le "politiquement correct»-, "sens et usages variés du vote» (le vote FN peut par exemple servir à des jeunes adultes à exprimer des intentions dans le but de s'opposer aux idées politiques «traditionnelles » de leurs parents), «trajectoires sociobiographiques » (le chercheur soutient notamment l'hypothèse qu'une partie des électeurs de Jean-Marie Le Pen votent pour lui car ils se reconnaissent dans le parcours social du personnage: adhésion à une idéologie de petit-bourgeois réactionnaire et désirs d'ascension sociale).

Dans sa conclusion, appelée ici «Épilogue », l'auteur résume sous la forme de "six traits majeurs ", les principaux points qu'il a développés dans son ouvrage afin de traiter du " phénomène Le Pen »: dynamique sociale complexe du vote $\mathrm{FN}$, multiplicité des intervenants s'exprimant sur la question, dénonciations morales, travail de construction symbolique, "effet d'offre " politique limité faisant du FN un "produit alternatif », usages sociaux variés du vote (pp. 95-98). Il se livre également en quelques 
lignes à une réflexion prospective sur l'avenir du FN (soulignant le moment problématique qui résultera de la "succession » du chef du parti). Enfin, évoquant une nouvelle fois le caractère discutable des discours produits sur la question, il conclut: " On peut néanmoins souhaiter que les agents sociaux aspirant à expliquer les votes se donnent les moyens conceptuels et empiriques de forger une grille explicative convaincante, c'est-à-dire qu'ils recentrent leurs investissements sur la fiabilité et la rigueur en s'éloignant du simplisme médiatique, du souci commercial, du conseil du prince, de la prescription thérapeutique et de la promotion disciplinaire» (p. 98). Notons que les dernières pages du livre sont consacrées à la présentation d'éléments factuels concernant Jean-Marie Le Pen et le Front national dans une perspective chronologique (pp. 99-114), ainsi qu'à une bibliographie d'une centaine de titres. Ces éléments complètent utilement le propos.

6 L'ouvrage de Jacques Le Bohec présente des dimensions intéressantes, heuristiques même, puisqu'à partir de l'objet qu'il traite, on comprend comment les biais affectant la réflexion intellectuelle sur le "phénomène Le Pen ", se retrouvent également lorsque l'on aborde d'autres «questions de société » donnant lieu à la production d'un discours médiatique surabondant. Ces éléments interrogent directement les sciences humaines et sociales et invitent à une réflexion sur leurs «limites »: comment peuvent-elles traiter de phénomènes aussi «polémiques » et "passionnés » que celui du vote FN ? En pareil cas, il importe que le scientifique puisse s'affranchir des conceptions communes et/ou médiatiques, ne se distinguant souvent pas de pré-notions et tous les passages du livre consacrés à la déconstruction de ces discours sont d'un grand intérêt, notamment pour un public d'étudiants. De même, la volonté de mettre à jour des explications multifactorielles pour expliquer le " phénomène » est à mettre au crédit de l'auteur.

Ce travail pose néanmoins un certain nombre de questions. La première tient à son titre: Sociologie du phénomène Le Pen. Si on apprend beaucoup de choses en lisant cet ouvrage, le sociologue restera toutefois quelque peu sur sa faim : sans doute de par le «format» de la publication, mais aussi, également, de par le propos développé par l'auteur, on passe un peu trop rapidement sur les éléments à proprement parler "sociologiques" du phénomène. Dans cette perspective, plutôt qu'une sorte de recension critique - et parfois même dénonciatrice - des travaux produits sur ce thème, les présentant souvent superficiellement, on aurait aimé bénéficier de davantage de matériaux empiriques permettant de mieux analyser ce fait social. L'autre question tient à cette accentuation sur la personne de Jean-Marie Le Pen telle qu'elle se pratique (dans les deux premiers chapitres notamment). Même si l'auteur s'explique sur ce positionnement et tente de le justifier (pp. 7-8), que force est de constater que les médias sont fascinés par ce "personnage ", on peut regretter, surtout si l'on reste fidèle à une perspective bourdieusienne, de ne pas en apprendre davantage sur les "agents sociaux» que sont les cadres du FN et ses militants, au profit d'une problématique recentrée sur le "chef ». Par exemple, selon nous, il aurait été utile de s'étendre davantage sur les différents groupes composant la mouvance du FN (catholiques intégristes, cercles ultralibéraux, voire adeptes du paganisme et des mythologies nordiques...) et son organisation interne, ses réseaux de militants. On pourra aussi s'étonner du ton familier, voire même badin, utilisé parfois par Jacques Le Bohec, qui n'est pas avare de jugements de valeur sur tel ou tel, de «bons mots » sur «Le Pen junior ». Ce point nuit à la crédibilité d'un propos se voulant scientifique. Dans le même registre, on pourra regretter que, dès les premières lignes, l'auteur se crôit obligé d'émettre des commentaires aux dimensions normatives, comme par exemple: 
«[...] il paraît absurde que l'« extrême droite " puisse encore avoir voix au chapitre » (p. 3), ou encore qu'il qualifie d'emblée le programme du FN de "régressif en matière de droits " (p.5: de «droits de la femme » si l'on comprend bien...). Quant au fait que «Dans la vie politique française de la fin $d u x^{e}$ et du début du xxI siècle, le phénomène Le Pen est sans doute l'événement le plus important » (p. 3), même si cette assertion justifie «l'accroche» de l'ouvrage, elle fait débat. Dans quelle mesure ce "phénomène» est-il plus important que la perte d'influence du PCF, les recompositions opérées au sein de la droite autour de l'UMP, les caractéristiques du vote écologiste, ou encore le fort taux d'abstentionnistes? Et si, au bout du compte, le FN n'était qu'un épiphénomène, parmi d'autres, de tendances plus lourdes encore mal étudiées par les sciences politiques?

\section{AUTEURS}

\section{LAURENT TRÉMEL}

Musée national de l'éducation, INRP 